\title{
Detection of Antimicrobial, Antioxidant and Cytotoxicity Activities of Fusarium oxysporum F01 Isolated from Catharanthus roseus Collected in Vietnam
}

\author{
Nguyen Hoang Khue Tu1,3* (D), Ngo Thi Thanh Thuy ${ }^{1,3}$ (D) Doan Thi Thanh Vinh ${ }^{1,3}$ (D), \\ Nguyen Pham Quynh Anh ${ }^{1,3}$ (D) and Ha Dieu Ly,3
}

${ }^{1}$ Department of Biotechnology, School of Biotechnology, International University, Ho Chi Minh City, Vietnam. ${ }^{2}$ School of Medicine, Vietnam National University, Ho Chi Minh City, Vietnam.

${ }^{3}$ Vietnam National University, Ho Chi Minh City, Vietnam.

\begin{abstract}
The ability of endophytic fungi to produce valuable bioactive compounds when surviving in medicinal herbs. Finding out endophytic fungi originated from Catharanthus roseus with antimicrobial, antioxidant and cytotoxicity activities is important for pharmaceutical development. The isolation was based on the morphology of fungi. Identification was performed by sequencing $18 \mathrm{~S}$ rRNA that was compared with known genes using Blast search in the combination of phylogenic analysis by using Clustal $\mathrm{W}$ and PhyML in GenomeNet. For the antimicrobial test, the agar diffusion method was used. DPPH scavenging assay for the antioxidant activity determination by using spectrophotometry. The cytotoxicity test was carried out by Sulforhodamine B method. LC-MS was applied for predicting components. We isolated the Fusarium oxysporum F01 strain originated from Catharanthus roseus. The aqueous extracts showed

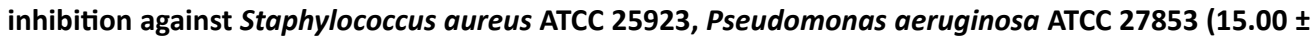
$0.50 \mathrm{~mm})$, Serratia marcescens ATCC $14756(9.00 \pm 0.87 \mathrm{~mm})$, Vibrio parahaemolyticus ATCC 17802 $(15.50 \pm 0.87 \mathrm{~mm})$, Escherichia coli ATCC $25922(12.50 \pm 0.50 \mathrm{~mm})$. The antioxidant activity of the extract obtained from the supernatant was determined with an IC50 value of about $11 \mu \mathrm{g} / \mathrm{mL}$. The extract also showed cytotoxicity effect on liver cancer cell line (HepG2) and breast cancer cell line (MCF-7) with
\end{abstract}

\footnotetext{
*Correspondence: nhktu@hcmiu.edu.vn; +84-2832744270
}

(Received: July 20, 2021; accepted: August 11, 2021)

Abbreviations: ANOVA: Analysis of variance, ATCC: American Type Culture Collection, BLAST: Basic Local Alignment Search Tool, DMEM: Dulbecco's Modified Eagle Medium, DPPH: 1,1-diphenyl-2-picrylhydrazyl, ETE: Environment for Tree Exploration, IC50: half maximal inhibitory concentration, LB: Luria-Bertani, LC-MS: Liquid chromatography mass spectroscopy, OD: Optical density PDB: Potato dextrose broth, SRB: Sulforhodamine B, TCA: trichloroacetic acid, YEME: Yeast Extract-Malt Extract. 
the percentage of inhibition of $84.47 \pm 3.18$ and $94.69 \pm 1.59$, respectively. LC-MS was used to point out the presence of pratol, a melanogenesis agent. The study provided more interesting information about Fusarium oxysporum F01 isolated in Catharanthus roseus grown in Vietnam, contributing to pharmaceutical sources in the world.

Keywords: Fusarium oxysporum F01, Catharanthus roseus, antimicrobial and antioxidant activity, cytotoxicity, LC-MS

\section{INTRODUCTION}

Microorganisms produced many biological compounds such as bacteriocin, conjugated linoleic acid, gamma buryric acid, lactic acid from lactic acid bacteria ${ }^{1-4}$, prodigiosin production from Streptomyces coelicolor ${ }^{5}$, penicillin from fungi ${ }^{6}$. Defined as an organism growing, existing within the plant causing no harm to its host, the endophyte is known to generate no apparent symptoms of disease and some of natural plant compounds ${ }^{7}$. Endophytic fungi are symbiotically associated with their host plant, mimic their chemistry and synthesize the same natural products and bioactive compounds as these originated from their host, and thus are screened for the production of valuable compounds. There were Taxol and taxane production by endophytic Taxomyces andreanae isolated from pacific yew ${ }^{8}$. Endophytic Cladosporium from Boerhaavia diffusa Linn showed in-vitro antioxidant activity9.

There were many plants showing biological activities such as Plumbago indica L. ${ }^{10}$, Alpinia conchigera Griff ${ }^{11}$, Achillea millefolium Linn $^{12}$, Murraya paniculata (L.) Bark ${ }^{13}$, Strobilanthes kunthianus ${ }^{14}$ and others. Catharanthus roseus or Vinca rosea, which is also commonly known as the Madagascar periwinkle or rose periwinkle is a plant species belongs to Apocynaceae family. It is used as an ornamental as well as medicinal plant. Catharanthus roseus is well known for production of copper nanoparticle ${ }^{15}$, antibacterial activities ${ }^{16}$, anti-cancerous agents such as vinca alkaloids which have effect on pain-relieving or contain anticancer properties. Vinblastine and vincristine of Catharanthus roseus have been developed and applied in anticancer drugs as prescriptions ${ }^{17}$. As a matter of fact, an endophyte called Fusarium oxysporum of rosy periwinkle plant, Catharanthus roseus was discovered to produce vincristine - an anticancer drug ${ }^{18-20}$. In term of Fusarium oxysporum, an ascomycete fungus of Nectriaceae family including many species that was discovered by Wollenweber and Reinking ${ }^{21}$. Fusarium oxysporum, which is dominant in active soil, can be harmless to plants or be a pathogenic to its host. In contrast, it is beneficial in form of endophytes - having a mutual relationship to plants, for example, some of these bioactive compounds have been found and extracted vincristine ${ }^{18-20}$. Moreover, antibiotic resistance in microorganisms is increasing with a high rate nowadays such as Salmonella sp. ${ }^{22}$, Escherichia coli and Klebsiella ${ }^{23}$, Staphylococcus aureus ${ }^{24}$. Additionally, antioxidant activities that can help human in preventing some diseases were studied much on plant extracts ${ }^{25-28}$ whereas microbial extracts were not investigated in both the depth and width.

Therefore, the study isolated Fusarium oxysprorum 01 from Catharanthus roseus collected in Binh Thuan (Vietnam). The strain was used to prove an excellent endophyte for many valuable substances. The antimicrobial assay was carried out to present the power of Fusarium oxysporum F01 isolated from Catharanthus roseus against Staphylococcus aureus ATCC 25923, Pseudomonas aeruginosa ATCC 27853, Serratia marcescens ATCC 14756, Vibrio parahaemolyticus ATCC 17802, Escherichia coli ATCC 25922 when using agar diffusion method and screened antioxidant activity and cytotoxicity. Moreover, the production of valuable compounds in a specific amount was also screened by liquid chromatography-mass spectrum (LC-MS). The results contributed to the evidence of endophytic fungi showing activities when having a mutual life with medicinal herbs.

\section{MATERIALS AND METHODS} Specimens

Catharanthus roseus trunk was collected in Binh Thuan (Vietnam).

Pathogenic bacteria including Staphylococcus aureus ATCC 25923, Pseudomonas aeruginosa ATCC 27853, Serratia marcescens ATCC 14756, Vibrio parahaemolyticus ATCC 17802, 
Escherichia coli ATCC 25922 were purchased from American typed culture Collection (USA).

Cancer cell lines (MCF-7 and Hep-G2) were purchased from Sigma (USA).

\section{Chemicals and reagents}

Culture media and medium components for media including Potato dextrose broth (PDB), Potato dextrose agar (PDA), Luria-Bertani (LB) broth, Yeast Extract, Malt Extract were purchased from Bio Basic (Canada).

1,1-diphenyl-2-picrylhydrazyl (DPPH) and Sulforhodamine B (SRB) were purchased from Sigma (USA).

Antibiotics were purchased from Institute of Drug Quality Control (Ho Chi Minh City, Vietnam).

DNA isolation kit was purchased from Qiagen (Germany).

Solvents were purchased from Merck (USA).

Other analytical chemicals were purchased from Thermo Fisher Scientific (USA).

\section{Isolation and Identification}

The trunk (100g) of Catharanthus roseus collected in Binh Thuan (Vietnam). Immediately, it was cut into small pieces, cultured in potato dextrose broth (PDB) in 5 days. The culture was spread on potato dextrose agar (PDA) to select the single colonies with mycelium. The colony was used to culture in PDB in 5 days for DNA extraction using DNA extraction kit. After checking the purity and concentration of DNA, DNA was used for sequencing. The sequence was compared to wellknown strains in gene bank using Blast search ${ }^{29}$. The sequence was aligned with the related sequence and reconstructed phylogenetic tree using the function "build" of the Environment for Tree Exploration (ETE3) v3.1.1 $1^{30}$ as implemented on the GenomeNet. ML tree was inferred using PhyML v20160115 ran with model and parameters according to Guidon et a ${ }^{31}$.

\section{Cultivation for antimicrobial activity}

Fusarium oxysporum F01 isolated from Catharanthus roseus in Vietnam was cultured in modified yeast extract- malt extract (YEME) as medium for the fermentation process in 14 days old cultures. The culture of Fusarium oxysporum F01 were centrifuged at $10,000 \mathrm{rpm}$ for 10 minutes at $25^{\circ} \mathrm{C}$ to separate the mycelia from the culture broth as pellet and supernatant. The supernatant was concentrated and fractionated with distilled water in an appropriate proportion for further analysis.

For the preparation crude pellet extract, the pellet $(1 \mathrm{~g})$ was sonicated in water before centrifugation. The crude pellet extract was collected for furthur analysis.

\section{Antimicrobial activity test}

Pathogenic organisms for testing were Staphylococcus aureus ATCC 25923, Pseudomonas aeruginosa ATCC 27853, Serratia marcescens ATCC 14756, Vibrio parahaemolyticus ATCC 17802, Escherichia coli ATCC 25922. From stock, the pathogens were inoculated and cultured in LB broth until OD600 of 0.5 was achieved for further tests. Antibiotics including ampicillin, ciprofloxacin, gentamycin were used as control.

Agar well diffusion technique was used to evaluate the antimicrobial activity of aqueous extracts. As described by previous study ${ }^{32}$, the LB agar plate was inoculated by spreading $20 \mu \mathrm{L}$ of pathogen culture onto the agar plate by sterile swabs, then four 5-mm diameter holes were aseptically punched by sterile tips and then $80 \mu \mathrm{L}$ extracts from supernatant and intracellular extract obt from pellet extracts was introduced to the wells. Antibiotics were used as positive controls whereas the negative control was modified YEME. The agar plates were incubated under $37^{\circ} \mathrm{C}$ and such antimicrobial agents diffused into the agar and inhibit the growth of microbes. After $10-12$ hour incubation, the inhibition zones around the wells were measured. Table 1 showed the reference and the concentration of antibiotics corresponding to type of organisms.

Similarly, the SE samples were used to screen for antimicrobial activity. The antimicrobial compounds diffused into the agar and inhibited microbial growth. Consequently, the inhibition growth zones were measured.

Antioxidant by 1,1-diphenyl-2picrylhydrazyl (DPPH) free radical scavenging test The radical scavenging activity of different concentration of fungal crude extracts was determined by using DPPH assay ${ }^{33}$. DPPH (1,1-diphenyl-2-picrylhydrazyl) solution was prepared by dissolving DPPH with methanol. The DPPH solution was kept out of light. The blank was 
set up with $189 \mu \mathrm{L}$ of methanol along with $9 \mu \mathrm{L}$ of DPPH. Ascorbic acid was used as standard sample which was prepared by diluting stock solution with distilled water into different concentrations $(1,2$, $3,4,5,6 \mu \mathrm{g} / \mathrm{mL})$. The working solution of extracts was prepared with different concentrations $(1,2.5$, $5,7.5,10,12.5 \mu \mathrm{g} / \mathrm{mL}$ ) with distilled water. Then, $9 \mu \mathrm{L}$ DPPH solution was mixed with $180 \mu \mathrm{L}$ of standard solution or diluted extracts. All mixtures were incubated in the dark at room temperature for 30 minutes. The absorbance of the mixture was then recorded at $517 \mathrm{~nm}$. The experiment was triplicate.

\section{Cytotoxicity test}

Sulforhodamine B (SRB) assay was a colorimetric method to determine sensitivity and cytotoxicity of a substance ${ }^{34}$. The anionic dye binds electrostatically with the positive-charged parts of the proteins. The amount of binding dye correlates with the number of total cellular proteins. The cells were cultured to attain $70-80 \%$ coverage of culture flasks and passed into 96-well plate with a density of 104 cells per well. The plates were incubated at $37^{\circ} \mathrm{C}$ in $5 \% \mathrm{CO} 2$ for 24 hours. Next, the medium of sample was added into the wells and incubated at $37^{\circ} \mathrm{C}$ in $5 \% \mathrm{CO} 2$ for 48 hours. Then, the cells were fixed with trichloroacetic acid (TCA) and stained with SRB $(0.2 \%)$ and left at room temperature $\left(25^{\circ} \mathrm{C}\right)$ for 5 to 20 minutes. After SRB was discarded, the cells were washed gently with acetic acid (1\%) for four times. Let the cells

Table 1. Organisms with corresponding positive controls

\begin{tabular}{|c|c|c|}
\hline Test organisms & $\begin{array}{c}\text { Positive } \\
\text { control }\end{array}$ & $\begin{array}{l}\text { Concen. } \\
(\mu \mathrm{g} / \mathrm{mL})\end{array}$ \\
\hline Staphylococcus aureus & Gentamycin & 64 \\
\hline Pseudomonas aeruginosa & Ciprofloxacin & 2 \\
\hline Serratia marcescens & Ciprofloxacin & 4 \\
\hline Vibrio parahaemolyticus & Ampicillin & 32 \\
\hline Escherichia coli & Ampicillin & 128 \\
\hline
\end{tabular}

air-dry at room temperature $\left(25^{\circ} \mathrm{C}\right)$ from 12 to 24 hours. The results were obtained by loading 200 $\mu \mathrm{L}$ tris base into each well, then shaking for 10 minutes until dissolved completely for measuring the optical density (OD) at $492 \mathrm{~nm}$ and $620 \mathrm{~nm}$. In the test, the positive control was camptothecin $(0.07 \mu \mathrm{g} / \mathrm{mL})$ and the negative control was DMEM (0.25\%).

\section{Liquid Chromatography - Mass Spectroscopy (LC-MS)}

The predicted compounds containing in samples were determined by LC-MS. Begin with the ionization, the ions were separated according to their mass to charge $(\mathrm{m} / \mathrm{z}$ ) ratio; followed by the transfer of ions to mass analyzer. Then, the spectra were recorded in the positive ionization mode.

\section{Statistical analysis}

In antimicrobial assay, data were reported and presented as mean \pm standard deviation. The statistic was analyzed by SPSS software and Microsoft Excel, especially Two-way ANOVA was used for checking significant difference between two factors: stains and treatments; then Tukey test was applied as Post Hoc test for multiple comparisons of groups on treatment factor.

\section{RESULTS \\ Identification of fungi}

By using Blast search, the strain had $100 \%$ identity to Fusarium oxysporum strain F-H.6.5-030318-02 by comparing its $18 \mathrm{~S}, 5.8 \mathrm{~S}, 28 \mathrm{~S}$

Table 2. Inhibition zone diameter of water extracts to organisms

\begin{tabular}{lcc}
\hline Test organisms & $\begin{array}{c}\text { Positive } \\
\text { control }\end{array}$ & $\begin{array}{c}\text { Aqueous } \\
\text { extract }\end{array}$ \\
\hline Staphylococcus aureus & $18.50 \pm 0.50$ & $17.83 \pm 0.58$ \\
Pseudomonas aeruginosa & $30.33 \pm 0.58$ & $15.00 \pm 0.50$ \\
Serratia marcescens & $40.33 \pm 0.29$ & $9.00 \pm 0.87$ \\
Vibrio parahaemolyticus & $44.50 \pm 0.50$ & $15.50 \pm 0.87$ \\
Escherichia coli & $22.33 \pm 0.58$ & $12.50 \pm 0.50$ \\
\hline
\end{tabular}

Table 3. Cytotoxicity (\% inhibition) of the crude extract Fusarium oxysporum

\begin{tabular}{lccc}
\hline Cancer cell lines & Fungal extract & $\begin{array}{c}\text { Camptothecin } \\
\text { (Positive control) }\end{array}$ & $\begin{array}{c}\text { DMEM medium } \\
\text { (Negative control) }\end{array}$ \\
\hline Hep G2 & $84.47 \pm 3.18$ & $98.15 \pm 2.05$ & 0 \\
MCF-7 & $94.69 \pm 1.59$ & $97.62 \pm 2.80$ & 0 \\
\hline \hline
\end{tabular}


ribosomal RNA sequences to partial gene of $18 \mathrm{~S}$ ribosomal RNA at the internal transcribed spacer 1 , and complete sequence of $5.8 \mathrm{~S}$ ribosomal
RNA gene with the internal transcribed spacer 2. The sequence was deposited in DNA Data Bank of Japan (DDBJ) with accession number

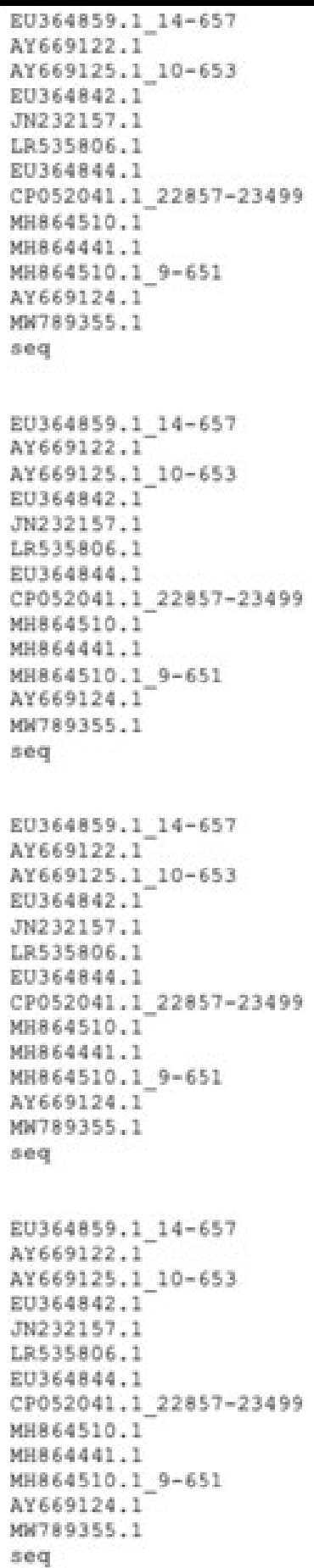

AACCCTCAAGCACAGCTYGGTGTGGGACTCGCGTYAATCGCGTTCCTC AACCCTCAAGCACAGCTTGGTGTTGGGACTCGCGTPAATTCGCGTTCCTC AACCCTCAAGCACAGCTRGGTGTGGGACTCGCGTTAATTCGCGTTCCTC AACCCTCAAGCACAGCTTGGTGTTGGGACTCGCGTTARTCGCGTTCCTC AACCCTCAAGCACAGCTTGGTGTTGGGACTCGCGTIAATTCGCGRTCCTC AACCCTCAAGCACAGCTTGGTGTTGGGACTCGCGTYARTCGCGTTCCCC AACCCTCAAGCACAGCTIGGTGTYGGGACTCGCGTTARTCGCGTTCCCC AACCCTCAAGCACAGCTTGGTGTTGGGACTCGCGTTAATTCGCGTTCCTC AACCCRCAAGCACAGCTTGGTGTTGGGACRCGCGTRAATCGCGTTCCTC AACCCRCAAGCACAGCTRGGTGTRGGGACRCGCGTAATTCGCGRTCCTC AACCCRCAAGCACAGCTRGGTGTRGGGACTCGCGTZARTCGCGTTCCTC AACCCRCAAGCACAGCTTGGTGTRGGGACRCGCGTRAATCGCGRTCCCC AACCCRCAAGCACAGCTRGGTGTRGGGACRCGCGTRAATTCGCGRTCCTC GATCCCRCCGC-TGGTTCACCAACGGAGACCTTGTACGACTTTACTTC $* * * * * * * * * * *$

AAATGATTGGCGGTCACGTCGAGCTTCCATAGCGT-AGTAGTAAA-ACC AAATTGATTGGCGGTCACGTCGAGCTRCCATAGCGT-AGTAGTAAA-ACC AAATTGATTGCCGGTCACGTCGAGCTFCCATAGCGT-AGTAGTAAA-ACC AAATTGATTGGCGGTCACGTCGAGCITCCATAGCGT-AGTAGTAAA-ACC AAATTGATZGGCGGTCACGTCGAGCTRCCATAGCGT-AGTAGTAAA-ACC AAATTGATTGGCGGTCACGTCGAGCTTCCATAGCGI-AGTAGTAAA-ACC AAATTGATRGCCGGTCACGTCGAGCTRCCATAGCGZ-AGTAGTAAA-ACC AAATTGATTGGCGGTCACGTCGAGCTTCCATAGCGT-AGTAGTAAA-ACC AAATGATTGGCGGTCACGTCGAGCITCCATAGCGI-AGTAGTAAA-ACC AAATTGATTGGCGGTCACGTCGAGCTTCCATAGCGZ-AGTAGTAAA-ACC AAATGATTGGCGGTCACGTCGAGCTTCCATAGCGZ-AGTAGTAAA-ACC AAATTGATTGGCGGTCACGTCGAGCTTCCATAGCGZ-AGTAGTAAA-ACC AAATTGATRGGCGGTCACGTCGAGCTTCCATAGCGT-AGTAGTAAA-ACC CTCTAMATGACCGAGTTTGGAGAGCTTRCCGGCCCFGAGTGGTAGTTGCC

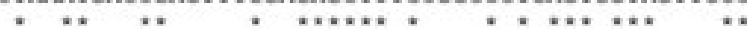

CTCGTYACTGGTAACGRCGCGGCCACGCCGTIAAACCCCAMCTTCTGAA CTCGIPACTGGTARCGICGCGGCCACGCCGTPAACCCCAACTICTGA CTCGTYACTGGTARCGTCGCGGCCACGCCGTTAAACCCCAACTPTGAA CTCGTIACRGGRARCGRCGCGGCCACGCCGTRAACCCCAMCTRCTGAA CTCGTTACrGGRAATCGRCGCGGCCACGCCGTYAAACCCCAACTTCTGAA CTCGTZACRGGRAARCGRCGCGGCCACGCCGTRAAACCCCAACRTCTGAA CTCGTPACRGGRAATCGRCGCGGCCACGCCGTRAAACCCCAACTRCTGAA CTCGTTACTGGTAATCGTCGCGGCCACGCCGTRAAACCCCAACTTCTGAA CTCGTTACTGGTAATCGRCGCGGCCACGCCGTRAAACCCCAACTTCRGAA CTCGTRACTGGTAARCGRCGCGGCCACGCCGTRAAACCCCAACTRCTGAA CTCGTYACTGGTAATCGTCGCGGCCACGCCGTYAAACCCCAACTRCTGAA CTCGTRACTGGTAATCGTCGCGGCCACGCCGTRAAACCCCAACTRCTGAA CTCGTTACrGGRAATCGRCGCGGCCACGCCGTTAAACCCCAACTRCTGAA CACCTCTCTGG=-GCCAGTCCGGACGCCTCACTGAGCC--ATTCAATCGG $* * * * * * * * * * * * * * * * *$

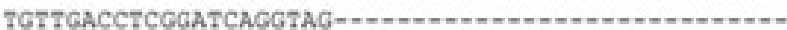

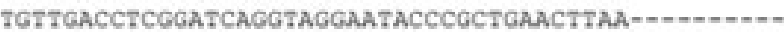
7GTZGACCTCCGATCAGGTAG-.... TGTTGACCTCGGATCAGGTAGGAATACCCGCTGAACTTAAG-m-m-m

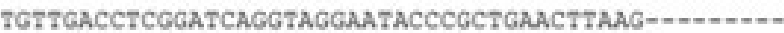

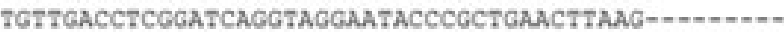

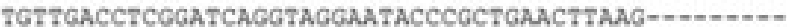
7GTтCACCTCGGATCACGTAGFGTTGACCRCGGATCAGGTAGGAATACCCGCTGACTTAAGCATATCAATGTTGACCTCGGATCAGGTAGGAATACCCGCTGAACTTAAGCATATCAAT TGTTGACCTCGGATCAGGTAG-. TGTTGACCTCGGATCAGGTAGGAATACCCGCTGAACTAA-..-....-TGTTGACCTCGGATCAGGTAGGAATACCCGCTGAACTTAAGCATATCAAT TAGTAGCGACGGGCGGTGT_..... 


\begin{tabular}{|c|c|}
\hline $\begin{array}{l}\text { EU364859.1.14-657 } \\
\text { AY669122.1 }\end{array}$ & 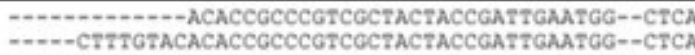 \\
\hline AY $669125.1 \quad 10-653$ & - ACACCGCCCGTCGCTACrACCGATTGAATGG--CT \\
\hline EU364842.1- & 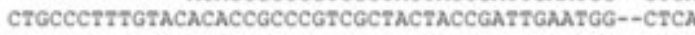 \\
\hline JN232157.1 & C2GCCCrTTGTACACACCGCCCGTCGCTACZACCGATTGAATGG--CI \\
\hline LR535806.1 & CTGCCCTтTGRACACACCGCCCGTCGCTACTACCGATTGAATGG--C2 \\
\hline E0364844.1 & CтGCCCTITGTACACACCGCCCGTCGCTACTACCGATTGAATGG--CI \\
\hline CP052041.1 22857-23499 & 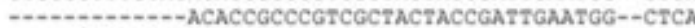 \\
\hline MH864510.1 & 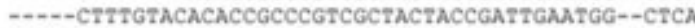 \\
\hline MH864441.1 & 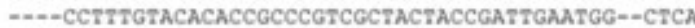 \\
\hline МH864510.1 9-651 & ACACCGCCCGTCGCTACTACCGATTGAATGG--C? \\
\hline AY $669124.1^{-}$ & -...-GCTACACACCGCCCGTCGCTACrACCGATTGAATGG--C2 \\
\hline MW789355.1 & - TGTACACACCGCCCGTCGCTACTACCGARTGAATGG--CT \\
\hline \multirow[t]{2}{*}{$\operatorname{seq}$} & W-NACCTGATCCGAGGTCAACATRCAGAATTGGGG \\
\hline & \\
\hline
\end{tabular}

EU364859.1_14-657

AY669122.1

AY $669125.1 \quad 10-653$

EU364842.1

JN232157.1

LR5 35806.1

EU364844.1

CP052041.1.22.857-23499

Mas 64510.1

MHB 64441.1

MH864510.1_9-651

AY669124.1

ME7 789355,1

seq

EU364859.2 14-65?

AY669122.1

AY669125.1 10-653

E0364842.1

JN232157.1

LR5 35806.1

EU3 364844.1

CP052041.1 22857-23499

MH8 64510.1

MH8 64441.1

MH864510.1_9-651

AY 669124.1

MW789355.1

seq

80364859.1 14-657

AY669122.1

AY669125.1_10-653

EU364842.1

JN232157.1

LR535806.1

EU364844.1

CP052041.1 22857-23499

MH864510.1

MHE 64441.1

NH864510.1_9-651

AY669124.1

MW789355.1

seq

E0364859.1_14-657

AY669122.1

AY669125.1_10-653

80364842.1

JN232157.1

LR5 35806.1

RU364844.1

CP052041.1 22857-23499

MH864510.1

KH864441.1

XH864510.1 9-65:

AY6.69124.1

MW789355.1

seq
GTGAGGCGTCCGGACTGGC--CCAGAGAGGTGGGCAACTACCACTCAGGG GRGAGGCGTCCGGACTGGC--CCAGAGAGGRGGGCAACTACCACTCAGGG GTGAGGCGTCCGGACTGGC--CCAGAGAGGTGGGCAACTACCACTCAGGG GrGACGCOTCCGGACFCGC--CCAGAGAGGRGGGCAACTACCACTCAGGG GTCAGGCGTCCGGACTCGC--CCAGACAGGICGGCAACTACCACTCAGGC GTGAGGCGTCCGGACTGGC--CCAGAGAGGTGGGCAACTACCACTCAGGG GTGAGGCGFCCCGACTGGC--CCAGAGAGGTGGGCAACTACCACTCAGGG GTCACCCOTCCCGACTOCC--CCAGAGACGTOCGCAACTACCACTCAGGG GTGACCCCTCCCGACFCGC--CCAGACAGGTCGGCAACTACCACTCAGCO GTCACGCGTCCGGACTGGC--CCAGAGACGRCGGCAACTACCACTCAGGO GZGAGGCGICCGGACTCGC--CCAGAGAGGZGGGCAACTACCACTCAGGO GFGAGGCOTCCGGACTOGC--CCAGAGAGGTCGGCAACTACCACTCAGGO GTGAGCCOTCCGGACZGGC--CCAGAGAGGTCGGCAACTACCACTCAGGO ACGGCGTGGCCGCGACGATTACCAGTAACGAGGGTT-TTACTACT-ACGC $,+, \ldots,+\ldots, \ldots, \ldots, \ldots, \cdots$

CCGGAAMGCTCTCCANACTCGGTCATTTAGAGGAAGTAMAGTCGTAACA CCGGAMGCFCTCCANACTCGGTCATTTAGAGGNAGTAMAGTCGTACA CCGGAAAGCFCTCCANACFCGGTCATTTAGAGGAAGTAANAGTCGTAACA CCGGAMACTCTCCANACTCGGTCATTIAGAGGAMGTAMAMGTCGAACA CCGGAARGCFCTCCAAACFCGGTCATTTAGAGGAAGTAAAAGCGTAACA CCGGAAAGCTCTCCAAACTCGGTCATTYAGAGGAAGTAAAGTCGTAACA СССGAMGCFCTCCAMACTCGGTCATTTAGAGGAAGTAMAGTCGTAACA CCGGAAAGCTCTCCAAACTCGGTCATTTAGAGGAAGTAAAAGTCGTAACA CCGGAAAGCTCTCCAAACTCGGTCATTRAGAGGAAGTAAAATCGTAACA CCGGAAAGCTCTCCAAACTCGGTCATTYAGAGGAAGTAAAAGTCGTAACA CCGGAAAGCTCTCCAAACTCGGTCATTTAGAGGAAGTAAAAGTCGTAACA ССGGAAAGCTCTCCAAACTCGGTCATTTAGAGGAAGTAMAGGCGTACA CCGGAAAGCTCTCCAAACRCGGTCATTTAGAGGAAGTAAAAGTCGTAACA TATGGAAGCFCGACGTGACCGCCAATCAATTTGAGGAACGCGAATTAACG , $\ldots \ldots,+\ldots,+, *,+, \ldots$

AGGTCTCCGRTGGTGAACCA-GCGGAGGGATCARTACCGAGTTACAAC2 AGGTCTCCOTTCGTGAACCA-GCGGAGGCATCATTACCGAGTTACAACT AGGFCFCCGTFGGTGAACCA-GCGGAGGOATCATZACCGAOTFTACAACT AGGTCTCCGFTGGTCAACCA-GCGGAGGGAZCATTACCGAGTTACAACT AGGICTCCGRTGGTCAACCA-GCGGAGGGAZCAZTACCGAGTZTACAAC? AGGTCTCCGTTCOTGAACCA-GCGGAGGCATCATTACCGAGTTTACAACT AGGTCTCCGTTGGTGAACCA-GCGGAGGGATCATTACCGAOTZTACAACT AGGTCTCCGTTGGTCAACCA-GCGGAGGOATCATTACCGAGTTACAACT AGOTCTCCGTTGGTCAACCA-GCGGAGGCATCATTACCGAGTTTACAACT AGGFCTCCGFTGGTGAACCA-GCGGAGGGATCATTACCGAGTFACAACT AGGTCTCCGTTCGTCAACCA-GCGGAGGGATCATTACCGAGTTACAACT AGGTCTCCGTTGGTGAACCA-GCGGAGGGATCATTACCGAGTTTACAACT AGGTCTCCGTTGGTGAACCA-GCGGAGGGATCATTACCGAGTTACAACT CGMGTCCCAACACCARGCTGTGCTTGAGGGTTGAMATGACGCTCG-AACA

* "* * ** * * * * "

CCCAAACCCCTGTGAACATACCACTTGTTGCCTCGGCGGATCAGCCCGCT CCCAAACCCCTGTGAACATACCACrTGTTGCCTCGGCGGATCAGCCCGCT СССАAССССТGTCAACATACCACFTGRTGCCTCGGCGGATCAGCCCGCT CCCAAACCCCTGTGAACARACCACTTGYTGCCTCGGCGGATCAGCCCGCZ CCCAAACCCCTGTGAACATACCACTTGTTGCCTCGGCGGATCAGCCCGCT CCCAAACCCCTGTGAACATACCACTTGTTGCCTCGGCGGATCAGCCCGCT CССAAACCCCTGTGAACATACCACFTGTTGCCTCGGCGGATCAGCCCGCT

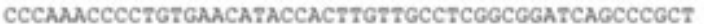
CCCAAACCCCTGTGAACARACCACTTGTTGCCTCGGCGGATCAGCCCGCT CCCAAACCCCTGTGAACATACCACTTGTTGCCTCGGCGGATCAGCCCGCT CCCAAACCCCTGTGAACATACCACTTGTTGCCTCGGCGGATCAGCCCGCT CCCAAACCCCRGTGAACATACCACRTGRTGCCTCGGCGGATCAGCCCGCT

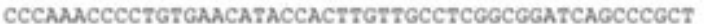
GOCATGCCC--GCCAGARACTGGCGGGCGCAATGTGCGTTCAAAATRC 
\begin{tabular}{ll}
\hline 0364859.1 & $14-657$
\end{tabular} AY 669122.1

AY 669125.1 10-653

gu364842.1

JN232157.]

LR. 535806.2

EU36.4844.1.

CP052041.1 22857-23499

мमㅛ64510.?

MH864441.

배864510.1 9-651

AY669124.1

Mex789355.1

seq

EU364859, $14-657$

AY669122.2

AY669125.1_10-653

BU364842.1

JN232157.1

LR5 535806.1

EU364844.1

CP052041.1 22857-23499

YH864510.3

MH864441.1

MH864510.1 9-651

AY669124.1-

MW789355.1

seq

EU364859, 1 14-657

AY669122.2

AY $669125.1210-653$

EU364842.1

JN232157.1

LRS35806.1

EU364844.1

CP052041.1 22857-23499

대864510.3

МH8 64441.1

MH8 64510.1 9-651

AY669124.1

MEx79935:

seq

EU364859.1.14-657

AY 669122.1

AY 669125.1 10-653

BU364842.1

JN232157.

LR5 35806.1

EU364844.2

CP052041.1 22857-23499

MH864510.1

Nar864441.

MH864510.1 9-65?

AY669124.1

Mor8935.

sec

80364859.1 $14-657$

AY669122.?

AY669125.1_10-653

Eu364842.1

JN232157.1

L.R535.806.

Eu364844.1

CP052041.1_22857-23499

MHB 64510.1

Nㅐ864441.2

MH8 64510.1 9-651

AY 669124.3

YW789355.2

sec

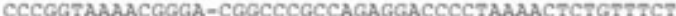

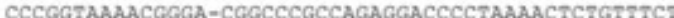
CCCGGTAAAACGGGA-CGGCCCGCCAGAGGACCCCTAAAACTCZGTYTC7 CCCGGTAAAACGGGA-CGGCCCGCCAGAGGACCCCTAAA-CTCTGTYTC: CCCGGTAAAACGGGA-CGCCCCGCCACAGGACCCCTAAA-CTCTGTTTCH CCCGGTAAAACGGGA-CGOCCCGCCAGAGGACCCCTAAA-CTCTGTTTC: СССGGTAAAACGGGA-CGGCCСGCCAGAGCACCCCTAAA-C7C7GTR7C? CCCGGTAAAACGGGA-CGGCCCGCCACAGCACCCCTAAA-CTCTCTRTC: CCCGGTAAAACGGGA-CGGCCCGCCAGAGGACCCCTAAA-CTCRGTITCI CCCGGTANAACGGGA-CGGCCCGCCAGAGGACCCCTAAA-CTCTGTITC: CCCGGTAAAACGGGA-CGGCCCGCCAGAGGACCCCIAAA-CTCTGTITC: CСCGGTAMAACGGGA-CGGCCCGCCACAGGACCCCTAAA-CTCTGTITC

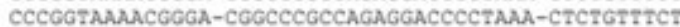

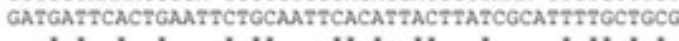

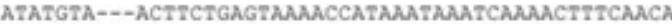

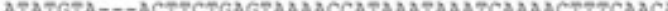
ATATGTA--ACTRCTGAGTAAACCATAAATAAATCAAAATTTCAACA ATATGTA--ACTrCTGAGTAAACCATAAATAAATCAגAATTTCAACA ATATGTA---ACTTCTGAGTAAAACCATAAAPAAATCARAATTTCAACA ATATGTA--ACTTCTGAGTAAAACCATAXATAAATCARAACTTTCAACA

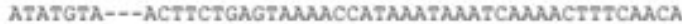

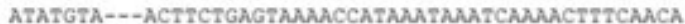
ATATGTA---ACTTCTGAGTAAAACCATARAYAAATCAAAACTTTCAACA ATATGTA---ACTPCTGAGTAAAACCATARATAAATCAAAACTTCAACA ATATGTA-NACTRCTGAGTAAAACCATAAATAAATCAAAACTRTCAACA ATATGTA--ACTTCTGAGTAAACCATAAATAARTCARACTTTCAACA ATATGTA-N-ACTTCTGAGTAAAACCATAAAZAAATCAAAACTZTCAACA TRCTRCATCGATGCCAGACCAAGAGATCCGZTGRTGAAATTRTGATT:

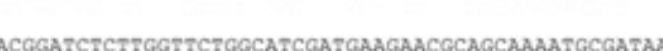

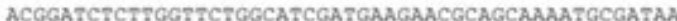
ACGGATCRCTYGGTYCTGGCATCGATGAAGAACGCAGCAAAATGCGATAA ACGGATCTCTRGGTCTGCCATCGATGAAGAACGCAGCAAAATGCGATAA ACGGATCFCTRGGYTCFGGCATCGATCAMGAACGCAGCAMAATGCGATA ACGGATCTCTYGGITCRGGCATCGATGAAGAACGCAGCANAATGCGATA ACGGATCRCTRGGTTCRGGCATCGATGAAGAACGCAGCANAATGCGATA ACGGATCTCTRGGTTCFCGCATCGATGAACAACGCAGCAMATGCGATA ACGGATCICTIGGITCRGGCATCGATGAAGAACGCAGCAAAATGCGATAA ACGGATCTCTYGGRTCRGGCATCGA GAAGAACGCAGCAAAATGCGATA ACGGATCRCTTGGTTCR GGCATCGATGAAGAACGCAGCAAATGCGATA ACGGATCTCTIGGITCRGGCATCGATGAAGAACGCAGCAMATGCGATA ACGGATCrCTrGGRTCRGCCATCGATCAAGAACGCAGCAMAATGCGAIAA ATTIATGGTTZACTCAGAAGT---TACATAZAGAACAGAGTZTAG-GG * * * ** * * * * * **

GTAATGTAATTCCAGAATCAGTGAATCATCGAATCTTTGAACGCACA GTAATGTGAATTCCAGAATZCAGTGAATCATCGAATCTTTGAACGCACA GPAATGTGAATTCCAGAATCAGTGAATCATCGMATCITTGACGCACA GTAARGTGAATTCCAGAATYCAGTGAATCATCGAATCITTGAACGCACA GTAATGTGAATTCAGAATCAGTGAATCATCGAATCTTYGACGCACA GTAATGTGAATGCAGAATCAGTGAATCATCGAATCTTTGAACCACA: GPAATGTGAATTGCAGAATTCAGTGAATCATCGAATCRTTGAACGCACA GTARGTGAATCCAGAATYCAGTGAATCATCGAATCZTTGAACGCACA2 GTARTTGAATTCCAGAATYCAGTGAATCATCGAATCZTRGAACGCACA: GTAATGTGAATTCACAATCAGTGAATCATCGAATCTTTGAACGCACA: GTAATGTGAATTCCAGAATCAGTGAATCATCGAATCZTTGAACOCACA GTAATGTGAATGCAGAATTCAGTGAATCATCGAATCZTTGAACGCACA GTARTGTGARTGCAGAATYCAGTGAATCATCGAATCFTTGAACGCACA GTCCTCTGGCGGGCCGT-CCCGTTRACCGGGAGCGGGCTGATCCGCCGA . . . . . ? . .

TGCGCCCGCCAGTATTCFGGC--GGGCATCCCTGRT-CGAGCGTCAFTTC TGCGCCCGCCAGTATTCZGGC--GGGCATGCCTGRZ-CGAGCGTCAITTC TGCGCCCGCCAGTATTCTGGC--GGGCAIGCCTGIT-CGAGCGTCAITIC TGCGCCCGCCAGTATTCTGGC--GGGCATGCCTGTT-CGAGCGTCAITTC TGCGCCCGCCAGTATTCTGGC--GGGCATCCCNGTZ-CGAGCGTCATTTC TGCGCCCGCCAGTATTCTGGC--GGGCATGCCTGIZ-CGAGCGRCAITTC TGCGCCCGCCAGTATTCTGGC--GGGCATCCCTGTI-CGAGCGTCATTRC TGCGCCCGCCAGTATTCTGGC--GGGCATGCCTGTT-CGAGCGTCATTYC TGCGCCCGCCAGTATICTGGC--GGGCATGCCTGIT-CGAGCGTCATTIC TGCGCCCGCCAGTATTCZCGC--GGGCATGCCTGIZ-CGAGCGTCAITIC TGCGCCCGCCAGTATTCTGGC--GGGCATGCCTGTT-CGAGCGTCATTTC TGCGCCCGCCAGTATTCTGGC--GGGCATGCCTGTT-CGAGCGTCATTTC TGCGCCCGCCAGTATTCTGGC--GGGCATGCCTGTT-CGAGCGTCATTPC GGCAACAAGTGGTATGTTCACAGGGGTTGGGAGTTGAAACTCGGTAAT 
Fig. 1. Multiple sequence alignment. (EU364859.1:14-657: Fusarium oxysporum strain F-X.1.7-030520-12; AY669122.1: Fusarium oxysporum strain F-W.6.2-030304; AY669125.1:10-653: Fusarium oxysporum strain F-X.1.7030520-12; EU364842.1: Fusarium oxysporum strain F-H.6.5-030318-02; JN232157.1: Fusarium oxysporum isolate 152; LR535806.1: Fusarium oxysporum 247; EU364844.1: Fusarium oxysporum strain F-H.6.5-030318-01; CP052041.1:22857-23499: Fusarium oxysporum Fo47 chromosome IV; MH864510.1: Fusarium oxysporum strain CBS 127297; MH864441.1: Fusarium oxysporum strain CBS 127149; MH864510.1:9-651: Fusarium oxysporum strain CBS 127297; AY669124.1: Fusarium oxysporum strain F-H.6.5-030318-J1; MW789355.1: Fusarium oxysporum isolate F3-2018; EU364842.1: Fusarium oxysporum strain F-H.6.5-030318-02; JN232157.1: Fusarium oxysporum isolate 152; AY669122.1: Fusarium oxysporum strain F-W.6.2-030304; LR535806.1: Fusarium oxysporum 247; seq: the sequence for isolated strain).

(LC645215). The sequence was aligned with the sequences of other Fusarium oxysporum strains (Fig. 1). Moreover, after phylogeny analysis using neighbor joining evaluation, the strain was closed to Fusarium oxysporum strains (Fig. 2). As a result, Fusarium oxysporum 01 originated from Catharanthus roseus was identified.

\section{Antimicrobial activity}

The antimicrobial activity of Fusarium oxysporum F01 on pathogens was demonstrated in Table 2. As shown in Table 2, the extract collected from supernatant of culture had effect on five organisms. The extract had the strongest inhibitory effect on Staphylococcus aureus (17.8 $\pm 0.58 \mathrm{~mm}$ )

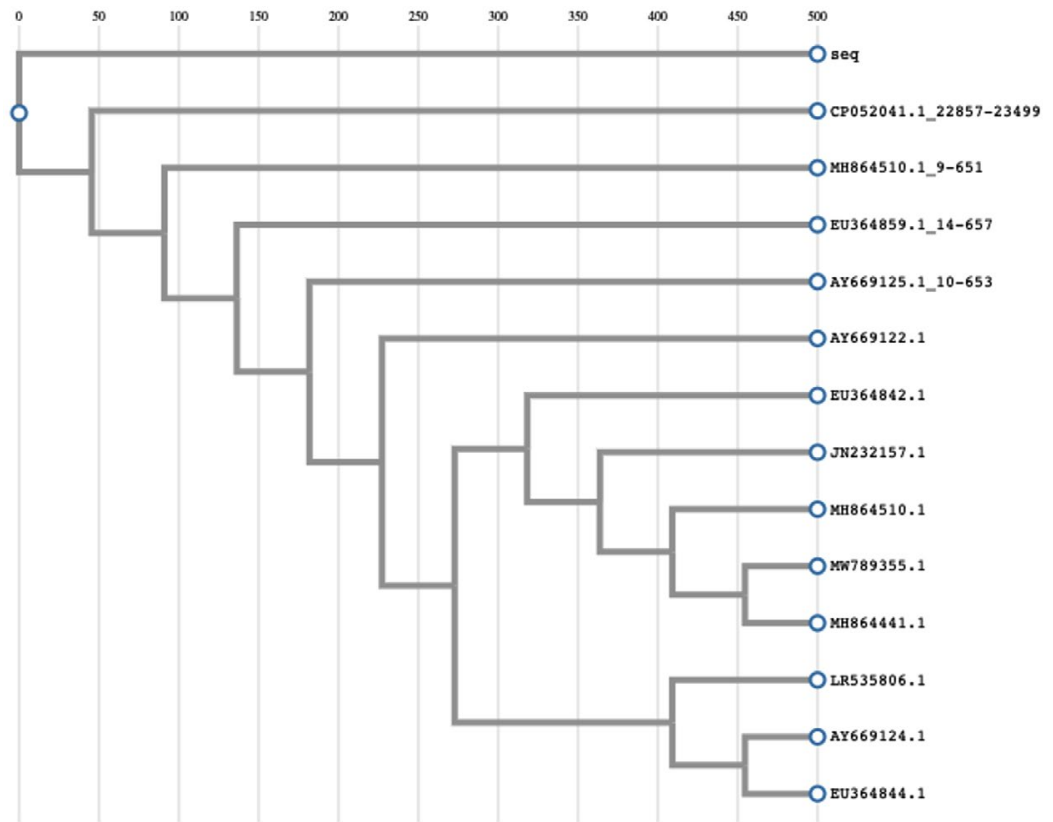

Fig. 2. Phylogeny analysis. (EU364859.1:14-657: Fusarium oxysporum strain F-X.1.7-030520-12; AY669122.1: Fusarium oxysporum strain F-W.6.2-030304; AY669125.1:10-653: Fusarium oxysporum strain F-X.1.7-030520-12; EU364842.1: Fusarium oxysporum strain F-H.6.5-030318-02; JN232157.1: Fusarium oxysporum isolate 152; LR535806.1: Fusarium oxysporum 247; EU364844.1: Fusarium oxysporum strain F-H.6.5-030318-01; CP052041.1:22857-23499: Fusarium oxysporum Fo47 chromosome IV; MH864510.1: Fusarium oxysporum strain CBS 127297; MH864441.1: Fusarium oxysporum strain CBS 127149; MH864510.1:9-651: Fusarium oxysporum strain CBS 127297; AY669124.1: Fusarium oxysporum strain F-H.6.5-030318-J1; MW789355.1: Fusarium oxysporum isolate F3-2018; EU364842.1: Fusarium oxysporum strain F-H.6.5-030318-02; JN232157.1: Fusarium oxysporum isolate 152; AY669122.1: Fusarium oxysporum strain F-W.6.2-030304; LR535806.1: Fusarium oxysporum 247; seq: the sequence for isolated strain). 
than Vibrio parahaemolyticus $(15.50 \pm 0.8 \mathrm{~mm})$ and Serratia marcescens $(9.00 \pm 0.87 \mathrm{~mm})$ and Escherichia coli (12.50 $\pm 0.50 \mathrm{~mm})$ Pseudomonas aeruginosa $(15.00 \pm 0.50 \mathrm{~mm})$. Specially, when the effect against Staphylococcus aureus was compared to Gentamycin $(64 \mu \mathrm{g} / \mathrm{mL})$, the extract gave inhibition zone that was not significant to gentamycin by statistical analysis. This fungus could be an ideal source of antimicrobial agent with high potency in Staphylococcus aureus treatment while Staphylococcus aureus is resistant to antibiotic highly.

For Pseudomonas aeruginosa, Serratia marcescens, Vibrio parahaemolyticus and Escherichia coli, the activities against these strains were not as strong as antibiotics used as control samples. However, the results showed the extract could contain many potential agents. To improve the activity, the concentration of extract should be increase. Moreover, all the extracts obtained from pellet did not show antimicrobial activity. To predict which compound in the pellet originated extract, LC-MS was presented in Fig. 3. The molecular mass $\left(\left[\mathrm{M}^{+} \mathrm{H}\right]^{+}\right)$was 269.0748 . The detection showed pratol in the extract. Pratol is an melanogenesis ${ }^{35}$. Therefore, the pellet extract did not show activities tested in the study.

Antioxidant by 1,1-diphenyl-2-picrylhydrazyl (DPPH) free radical scavenging

The evaluation of the antioxidant activity of the extract was determined by measuring its scavenging ability against DPPH in the stable radical assay. Based on the curve of ascorbic acid $\left(y=8.0796 x+39.646, r^{2}=0.9899\right)$, the antioxidant activities of the extract prepared with different concentrations were determined. All the inhibitory percentage in antioxidant assay built up the straight curve $\left(y=8.0796 x+39.646, r^{2}=0.9899\right)$ (Fig. 4). It meant that the obtained results were relative and reliable. The IC50 of crude extract is $11.03 \mu \mathrm{g} / \mathrm{mL}$ that was higher than ascorbic acid (IC50 $=8 \mu \mathrm{g} / \mathrm{mL}$ ). The crude extract showed the weaker antioxidant activity than ascorbic acid.

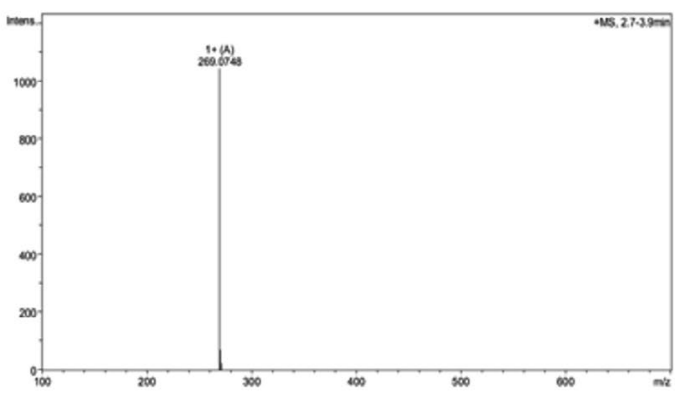

Fig. 3. LC-MS analysis of the extract obtained from pellet.

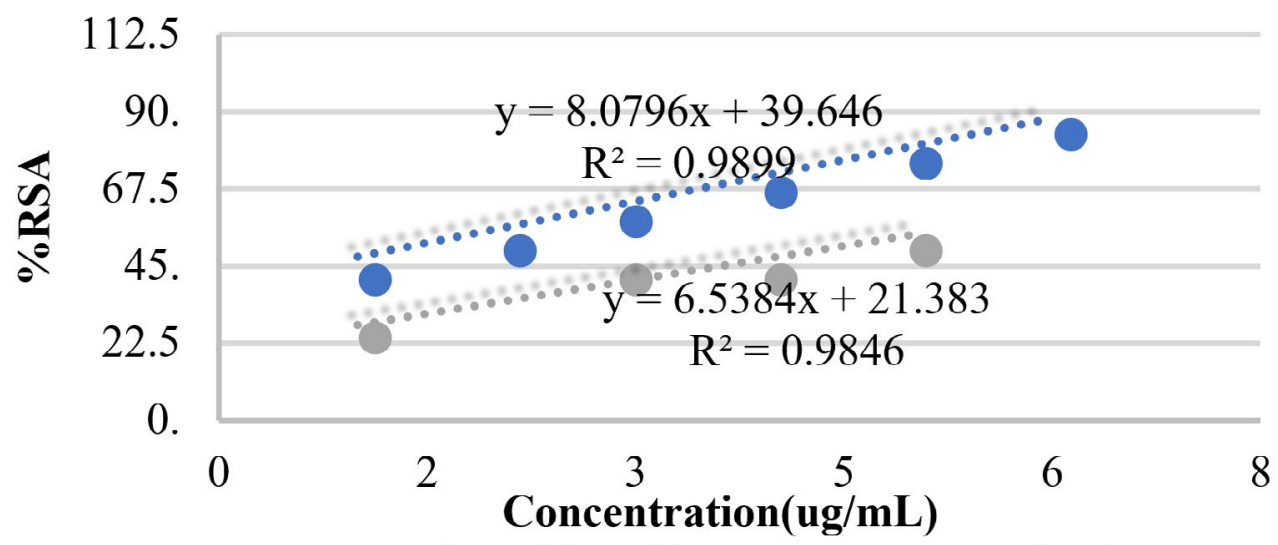

Ascorbic acid

Crude

Fig. 4. Percentage radical scavenging activity of ascorbic acid and crude extract. 


\section{Cytotoxicity activities}

The samples were prepared at the concentration of $50 \mathrm{mg} / \mathrm{mL}$ for cytotoxicity test on MCF-7 and Hep-G2 cells. All the samples had cytotoxicity on these cell line (Table 3 ). In the study, we couldn't detect vincristine and vinblastine in Fusarium oxysporum F01 by LC-MS. The cytotoxicity was caused by others compounds in Fusarium oxysporum F01.

The biological activities were due to this strain had symbiotic effects with Catharanthus roseus which had well-known activities. Fusarium oxysporum originated from environment commonly causes diseases, however, Fusarium oxysporum F01 showed biological activities such as antimicrobial and antioxidant activities together with cytotoxicity. The study brings out the importance of microoganisms showing pathogenic or beneficial effects depending on their host.

\section{DISCUSSION}

BLAST search is the tool for comparing biological sequence information. A BLAST search help to identify database sequences which are similarity to the unknown sequence. In the study, Fusarium oxysporum F01 had partial sequence of $18 \mathrm{~S}, 5.8 \mathrm{~S}$ and $28 \mathrm{~S}$ rRNA with $100 \%$ identity to the gene information of strain F-H.6.5030318-02. the reconstruction, analysis, and visualization of phylogenetic trees and multiple sequence alignments were analyzed simplify by ETE that supports in comparative genomics and phylogenetics. In the combination of phylogeny analysis, Fusarium oxysporum F01 originated from Catharanthus roseus was identified obviously.

Table 2 showed the antimicrobial activity of Fusarium oxysporum F01 extract collected from supernatant of culture on five organisms. The extract showed inhibition against the growth of Staphylococcus aureus, Vibrio parahaemolyticus, Serratia marcescens and Escherichia coli and Pseudomonas aeruginosa in different manners, leading to the different effects. Interestingly, the potency of the extract was not significant to gentamycin $(64 \mu \mathrm{g} / \mathrm{mL})$ by statistical analysis when inhibited Staphylococcus aureus growth. Therefore. Exploiting this strain metabolites to treat these bacteria is important to inhibit the tested bacteria that had risks of resistance such as Staphylococcus aureus. The extract obtained from cultural supernatant showed the weaker cytotoxicity activity than ascorbic acid due to it contained the mixture of antioxidant agents and non-antioxidant agents that interfere the activity. However, the antioxidant agent existed in the extract after antioxidant activity screening. Moreover, the extract at the concentration of $50 \mathrm{mg} / \mathrm{mL}$ for cytotoxicity test on MCF-7 and Hep-G2 cells. By the comparison to the controls, the extract gave the significantly lower activity than camptothecin on HepG2, but insignificant difference from camptothecin on MCF-7. In the study, we couldn't detect vincristine and vinblastine in Fusarium oxysporum F01 by LC-MS analysis due to low concentration or the unsuitable cultivation. The cytotoxicity was caused by others compounds in Fusarium oxysporum F01. The pellet extract could not show strong antimicrobial and antioxidant activities in the study. However, the pellet extract contained pratol that is a melanogenesis. Obviously, Fusarium oxysporum F01 isolated from Catharanthus roseus showed the benefit activities, strengthening that symbiotic endophyte could produce useful activities when it may be harmful when living in the other sources.

\section{CONCLUSION}

Extract from cultural supernatant of Fusarium oxysporum F01 had inhibitory activities against Staphylococcus aureus ATCC 25923, Pseudomonas aeruginosa ATCC 27853, Serratia marcescens ATCC 14756, Vibrio parahaemolyticus ATCC 17802, Escherichia coli ATCC 25922. Fusarium oxysporum F01 will contribute to develop antimicrobial activities against Pseudomonas aeruginosa, Serratia marcescens, Staphylococcus aureus, Vibrio parahaemolyticus and Escherichia coli when these strains were resistant to antibiotics besides antioxidant and cytotoxicity activities. The pellet extract was screened to predict pratol used for melanogenesis prevention although it did not show antimicrobial and antioxidant activities. The mechanisms and purified compounds will be exploited to understand well and apply for future. 


\section{ACKNOWLEDGMENTS}

We would like to thank to Vietnam National University, Ho Chi Minh City (VNU-HCM) for the support.

\section{CONFLICT OF INTEREST} conflict of interest.

The authors declare that there is no

\section{AUTHORS' CONTRIBUTIONS}

NHKT proposal investigator, ideas, design experiment. NHKT, NTTT, DTTV and HDL performed the experiment. NHKT, NTTT, DTTV and HDL performed the data analysis. NPQA design figures and tables. NHKT, NTTT and NPQA wrote the manuscript. All authors read and approved the manuscript for final publication.

\section{FUNDING}

This research is funded by Vietnam National University, Ho Chi Minh City (VNU-HCM) under grant number C2019-28-02.

\section{DATA AVAILABILITY}

The datasets generated during and/or analysed during the current study are available from the corresponding author on reasonable request.

\section{ETHICS STATEMENT}

Not applicable.

\section{REFERENCES}

1. Nghe D, Nguyen T. Characterization of antimicrobial activities of Pediococcus pentosaceus Vtcc-B-601. J Appl Pharm Sci. 2014;4(5):61-64. doi: 10.7324/ JAPS.2014.40511

2. Anh NPQ, Yen TTH, Vinh DTT, Tien TP, Dung NA, Khue NH. Study on the ability of producing of conjugated Linoleic acid of Lactobacillus fermentum A01 isolated from human digestive tract. Res J Pharm Technol. 2021;14(3):1319-1322. doi: 10.5958/0974360x.2021.00234.1

3. Thien Phuc C, Thanh Vinh DT, Khoa My T, et al. Study on conditions for Gama buryric acid (GABA) production in Lactobacillus fermentum A01 isolated from human. Res J Pharm Technol. 2021;14(4):2188-2190. doi: 10.52711/0974-360x.2021.00387

4. Nguyen HHH, Nguyen DA, Nguyen TKH. Biological activities of poly (Lactic acid) polymer produced from Lactobacillus rhamnosus PN04. Res J Pharm Technol. 2018;11(7):3057-3062. doi: 10.5958/0974360X.2018.00562.0

5. Thu TTM, Vinh DTT, Dung NA, Tu NHK. Effect of lactic acid produced by lactic acid bacteria on prodigiosin production from streptomyces coelicolor. Res J Pharm Technol. 2021;14(4):1953-1956. doi: 10.52711/0974$360 X .2021 .00345$

6. Vo G, Nguyen D, Nguyen T. Improvement of antibiotic production in fungi. Res J Pharm Technol. 2018;11(7):3227-3233. doi: 10.5958/0974360X.2018.00593.0

7. Prerana V, Kilingar NV. Enhanced production of an Anti-Cancer pigment from Bacillus endophyticus JUPR15: Single factor system Vs RSM. Res J Pharm Technol. 2021;14(1):153-161. doi: 10.5958/0974360x.2021.00027.5

8. Stierle A, Strobel G, Stierle D. Taxol and taxane production by Taxomyces andreanae, an endophytic fungus of Pacific yew. Science. 1993;260(5105):214216. doi: 10.1126/science. 8097061

9. Sundar RDV, Anitha KPGU, Arunachalam S. In vitro Antioxidant and Phytochemical analysis of crude extracts of endophytic fungi (Cladosporium sp) from Boerhaavia diffusa Linn. Res J Pharm Technol. 2021;14(1):202-206. doi: 10.5958/0974360x.2021.00035.4

10. Dibyajyoti S, Swati P. Cytotoxic Activity of Methanolic Extract of Plumbago indica L. (Family: Plumbaginaceae). Asian J Pharm Tech. 2012;2(2):59-61

11. Dibyajyoti S, Swati P. Cytotoxic Activity of Methanolic Extract of Alpinia conchigera Griff (Family: Zingiberaceae). Asian J Pharm Res. 2012;2(2):86-88.

12. Subramanian G, Meyyanathan SN, Gowramma $B$, Palanisamy DS. Liquid chromatography-mass spectrometric method for simultaneous estimation of apigenin and luteolin from Achillea millefolium Linn. . Asian J Res Chem. 2016;9(12):629-632. doi: 10.5958/0974-4150.2016.00086.9

13. Nowaz RN, Mostafizur MR, Farhad MH. Free Radical Scavenging Activity and Cytotoxic Potential of Crude Extractives of Murraya paniculata (L.) Bark. Res J Pharmacogn Phytochem. 2012;4(1):18-22

14. Balasubramaniam G, Sekar M, Badami S. In-vitro Antioxidant and Cytotoxic properties of Strobilanthes kunthianus. Res J Pharm Technol. 2021;14(5):25222528. doi: 10.52711/0974-360x.2021.00444

15. Chauhan R, Patel C, Panigrahi J. Greener approach for copper nanoparticles synthesis from Catharanthus roseus and Azadirachta indica leaf extract and their antibacterial and antioxidant activities. Asian J Res Pharm Sci. 2018;8(2):81-90. doi: 10.5958/22315659.2018.00016.4

16. Safhi MM, Ali M, Sivakumar SM, Jabeen A, Manohar YN. Antibacterial studies of catharanthus roseus of jazan province against the selected bacterial strains. Res J Pharm Technol. 2013;6(4):403-405

17. Zhang LB, Gou LH, Zeng SV. Preliminary study on the isolation of endophytic fungus of Catharanthus roseus and its fermentation to produce products of therapeutic value. Chin Tradit Herb Drugs. 2000;31(11):805-807

18. Tung CY, Yang DB, Gou M. A Preliminary study on the condition of the culture an isolate of endophytic fungus producing vincristine. J Chuxiong Norm Univ. 2002;6:39-41 
19. Kour A, Shawl AS, Rehman S, et al. Isolation and identification of an endophytic strain of Fusarium oxysporum producing podophyllotoxin from Juniperus recurva. World J Microbiol Biotechnol. 2008;24(7):1115-1121. doi: 10.1007/s11274-0079582-5

20. Shams KA, Nazif NM, Abdel Azim NS, et al. Isolation and characterization of antineoplastic alkaloids from Catharanthus roseus L. Don. cultivated in Egypt. African $J$ Tradit Complement Altern Med. 2009;6(2):118-122. doi: 10.4314/ajtcam.v6i2.57082

21. Wollenweber HW, Reinking OA. Die Fusarien, ihre Beschreibung, Schadwirkung und Bekämpfung. Paul Parey; 1935

22. Kanth SV, Aparna M, Sumit S, Jabez OW. Isolation and characterization of drug resistant Salmonella typhi from sewage water. Res J Pharm Technol. 2015;8(2):167-171. doi: 10.5958/0974-360X.2015.00030.X

23. Abbas HA, Kadry AA, Shaker GH, Goda RM. Resistance of Escherichia coli and Klebsiella pneumoniae isolated from different Sources to $\beta$-lactam Antibiotics. Res J Pharm Technol. 2017;10(2):589-591. doi: 10.5958/0974-360x.2017.00116.0

24. Bharadwaj, Gopinath P. Detection of inducible clindamycin resistance among clinical isolates of Staphylococcus aureus. Res J Pharm Technol. 2018;11(9):3990-3992. doi: 10.5958/0974360X.2018.00733.3

25. Sarkar D, Debnath S, Bose SK. Phytochemical analysis and assessment of Antioxidant properties of black tea extract obtained from Camellia sinensis. Res J Pharm Technol. 2020;13(10):4539-4544. doi: 10.5958/0974360x.2020.00800.8

26. Keshri V, Dutt KR. Inhibitory effect of phenolic and flavonoidal content of $\mathrm{H}$. indicum root extract on 1, 1-diphenyl-2-picrylhydrazyl radicals. Res J Pharm Technol. 2021;14(1):235-238. doi: 10.5958/0974-360x.2021.00041.x
27. Mohamed SH, Mohamed WS, Shaheen MNF, Elmahdy EM, Mabrouk MI. Cytotoxicity, Antibiotic Combination and Antiviral Activity of Papain Enzyme: In vitro study. Asian J Res Pharm Sci. 2020;10(1):6-10. doi:10.5958/2231-5659.2020.00002.8

28. Soni A, Femida P, Sharma P. In-vitro cytotoxic activity of plant saponin extracts on breast cancer cell-line. Res J Pharmacogn Phytochem. 2017;9(1):17-22. doi: 10.5958/0975-4385.2017.00003.6

29. Altschul SF, Madden TL, Schaffer AA, et al. Gapped BLAST and PSI-BLAST: A new generation of protein database search programs. Nucleic Acids Res. 1997;25(17):3389-3402. doi: 10.1093/nar/25.17.3389

30. Huerta-Cepas J, Serra F, Bork P. ETE 3: Reconstruction, Analysis, and Visualization of Phylogenomic Data. Mol Biol Evol. 2016;33(6):1635-1638. doi: 10.1093/ $\mathrm{molbev/msw046}$

31. Guindon S, Dufayard JF, Lefort V, Anisimova M, Hordijk W, Gascuel O. New algorithms and methods to estimate maximum-likelihood phylogenies: Assessing the performance of PhyML 3.0. Syst Biol. 2010;59(3):307-321. doi: 10.1093/sysbio/syq010

32. Rajesh T, Venkatanagaraju E, Goli D, Basha SJ. Evaluation of antimicrobial activity of different herbal plant extracts. Int J Pharm Sci Res. 2014;5(4):14601468. doi: 10.13040/IJPSR.0975-8232.5(4).1460-68

33. Brand-Williams W, Cuvelier ME, Berset C. Use of a free radical method to evaluate antioxidant activity. $L W T$ - Food Sci Technol. 1995;28(1):25-30. doi: 10.1016/ S0023-6438(95)80008-5

34. Skehan P, Storeng R, Scudiero D, et al. New colorimetric cytotoxicity assay for anticancer-drug screening. J Natl Cancer Inst. 1990;82:1107-1112. doi: 10.1093/ jnci/82.13.1107

35. Chung YC, Kim S, Kim JH, et al. Pratol, an O-methylated flavone, induces melanogenesis in B16F10 melanoma cells via $\mathrm{p}-\mathrm{p} 38$ and $\mathrm{p}$-JNK upregulation. Molecules. 2017;22(10):1704. doi: 10.3390/molecules22101704 